\title{
Lactate kinetics in sepsis and septic shock: a review of the literature and rationale for further research
}

\author{
Jason Chertoff ${ }^{*}$, Michael Chisum, Bryan Garcia ${ }^{2}$ and Jorge Lascano ${ }^{3}$
}

\begin{abstract}
Over the last two decades, there have been vast improvements in sepsis-related outcomes, largely resulting from the widespread adoption of aggressive fluid resuscitation and infection control. With increased understanding of the pathophysiology of sepsis, novel diagnostics and resuscitative interventions are being discovered. In recent years, few diagnostic tests like lactate have engendered more attention and research in the sepsis arena. Studies highlighting lactate's prognostic potential for mortality and other outcomes are ubiquitous and largely focus on the early stage of sepsis management, defined as the initial $6 \mathrm{~h}$ and widely referred to as the "golden hours." Additional investigations, although more representative of surgical and trauma patients, suggest that lactate measurements beyond $24 \mathrm{~h}$ from the initiation of resuscitation continue to have predictive and prognostic utility. This review summarizes the current research and evidence regarding lactate's utility as a prognosticator of clinical outcomes in both early and late sepsis management, defines the mechanism of lactate production and clearance, and identifies areas warranting further research.
\end{abstract}

Keywords: Lactate, Kinetics, Sepsis, Early sepsis, Late sepsis, Resuscitation, Microvascular, Shock

\section{Introduction}

Nearly a decade since the landmark article, "Surviving Sepsis Campaign Guidelines for Management of Severe Sepsis and Septic Shock," sepsis remains a hotbed of research, and new diagnostic and resuscitative interventions are under continual development and evaluation. One such effort that has received focus is the role of lactate monitoring and whether measurements taken on initial presentation and serially can be used as targets of clinical end points including adequate resuscitation and in-hospital mortality [1-6].

\section{Review}

Mechanism of hyperlactemia in sepsis and septic shock Traditionally, lactic acidosis in sepsis is attributed to anaerobic glycolysis due to inadequate oxygen delivery. However, it has become clear that the mechanism of hyperlactemia in sepsis is multifactorial and due to factors

\footnotetext{
* Correspondence: jason.chertoff@medicine.ufl.edu

'Department of Medicine, University of Florida College of Medicine, 1600 SW Archer Road, Gainesville, FL 32608, USA

Full list of author information is available at the end of the article
}

beyond hypoxic tissue injury alone [5-9]. For example, James et al. proposed that lactic acidosis refractory to standard resuscitation is frequently due to increased aerobic glycolysis in skeletal muscle secondary to epinephrine-stimulated $\mathrm{Na}^{+}, \mathrm{K}^{+}$-ATPase activity and not to anaerobic glycolysis from hypoperfusion, and warned that continued attempts at resuscitation targeting lactate levels could lead to unnecessary blood transfusion and use of inotropic agents [8]. Furthermore, Gutierrez et al. emphasized that the etiology of prolonged lactic acidosis in sepsis is often multifactorial, making it an unreliable marker of oxygen debt and inadequate resuscitation [7]. Interestingly, it has been demonstrated that septic patients with hyperlactemia after $24 \mathrm{~h}$ of resuscitation had similar lactate production but lower lactate clearance than septic patients with normal blood lactate [9]. This finding raises doubts about the reliability of hyperlactemia as an indicator of the intensity of anaerobic metabolism in septic patients, suggesting instead that persistence of hyperlactemia during sepsis may be more representative of 
inadequate lactate clearance as opposed to pure lactate overproduction [9].

These findings questioned the notion that hyperlactemia in sepsis and septic shock is solely the result of hypoperfusion. Further support for the role of inadequate lactate clearance stems from Gibot et al. who used an endotoxemia model to demonstrate that lactate levels in sepsis can be elevated despite adequate systemic perfusion, blood pressure, and oxygen delivery [10]. Similarly, in a pig model, Ven Genderen et al. showed that septic shock behaves differently from obstructive/circulatory shock that even when cardiac output and other systemic parameters are optimized, there continues to be regional microvascular oxygen mismatch in septic shock, as compared to obstructive/circulatory shock. The authors postulate that this regional microvascular oxygen mismatch leads to elevations in lactate that may be partially unresponsive to traditional resuscitative interventions that only target systemic parameters [11]. Likewise, Hernandez et al. showed lactate in septic shock to have a biphasic response, with an initial rapid improvement followed by a much slower normalization many hours later, and hypothesized that this slow and delayed response might be attributed to the microvascular oxygen mismatch as described by Ven Genderen et al., making further systemic resuscitation through traditional sepsis resuscitation bundles ineffective and perhaps detrimental [12]. In fact, Marik et al. attribute hyperlactemia in the later phase of sepsis to increased aerobic glycolysis due to a stress response and also label any attempts at using traditional sepsis therapies to normalize lactate during this later stage as flawed and potentially harmful [13]. With these findings, Rivers et al. warn against using only lactate clearance as a marker of sepsis recovery and state that lactate clearance, central venous oxygen saturation $\left(\mathrm{ScvO}_{2}\right)$, and other markers are complementary and not mutually exclusive end points [14].

Thus, new insight regarding the mechanism for hyperlactemia in sepsis following adequate initial resuscitation and infection control demonstrates that tissue hypoxia is not the sole etiology of hyperlactemia during late sepsis. Nevertheless, data supporting the clinical utility of lactate as a marker of early sepsis recovery is robust while the role of continued lactate monitoring beyond the initial resuscitation period into the stage of late sepsis and its potential to guide treatment during this later stage remains uncertain [15-18].

\section{Lactate as a prognosticator in early sepsis management}

In their 2004 and 2008 sepsis guidelines, Dellinger et al. recommended measurement of lactate on initial presentation, with an elevated value signifying tissue hypoperfusion and necessitating aggressive resuscitation [19-21].
Although their guidelines suggested measuring lactate only upon presentation, many clinicians and researchers have attempted to capitalize on the test's theoretical diagnostic and predictive value by including additional measurements during the resuscitation process [22-26]. For example, it was shown that lactate clearance greater than $10 \%$ from initial measurement during the first 2 to $6 \mathrm{~h}$ of resuscitation predicted survival from septic shock and that protocols targeting lactate clearance of at least $10 \%$ produced similar short-term survival rates to protocols using $\mathrm{ScvO}_{2}$ monitoring $[2,22,23,25]$. Moreover, it was demonstrated that for every $10 \%$ increase in lactate clearance, there was a corresponding $11 \%$ decrease in inhospital mortality [2]. Similarly, septic patients with lactate clearance of greater than $20 \%$ during the initial $8 \mathrm{~h}$ of resuscitation had a $22 \%$ decline in the relative risk of mortality, compared with patients having lactate clearances of less than $20 \%$ [24].

Since these initial studies are evaluating lactate as a marker of recovery in sepsis and septic shock, further research has evaluated the role of lactate monitoring during the early resuscitative period. For example, Puskarich et al. studied resuscitation during the initial $6 \mathrm{~h}$ of treatment and demonstrated that achieving an $\mathrm{ScvO}_{2}$ goal $\geq 70 \%$ without obtaining a lactate clearance goal $\geq 10 \%$ was associated with higher mortality than reaching the lactate clearance goal without the $\mathrm{ScvO}_{2}$ goal [27]. Furthermore, these same authors showed that early lactate normalization (within $6 \mathrm{~h}$ ) was a predictor of survival in patients being treated for sepsis and septic shock [28]. Nguyen et al. investigated the addition of lactate clearance within the first $12 \mathrm{~h}$ of resuscitation to the severe sepsis resuscitation bundle and showed that including lactate clearance leads to an almost twofold increase in relative risk reduction of death [29]. In response to the convincing literature supporting the utility of lactate clearance in early sepsis, the newest surviving sepsis guidelines for early goal-directed therapy (EGDT) now includes lactate clearance during the first $6 \mathrm{~h}$ of resuscitation as a goal of early resuscitation [24]. Thus, research regarding lactate monitoring as a marker of recovery in severe sepsis and septic shock has proven fruitful but has primarily focused on the early resuscitation period [23-28].

\section{Lactate as a late prognosticator in sepsis management}

Literature evaluating the clinical and predictive value of lactate measurements beyond the initial 6 -h resuscitation period in the medical management of sepsis is significantly less robust [30-34]. In a study of 137 surgical intensive care unit (SICU) patients, Husain et al. showed elevated initial and 24-h lactate levels to be significant predictors of mortality, with mortality ranging from 10 to $67 \%$ depending on whether lactate levels normalized 
or failed to normalize at $24 \mathrm{~h}$, respectively [35]. In another study investigating SICU patients, Bakker et al. showed that lactate clearance measured $24 \mathrm{~h}$ after admission was a significant predictor of in-hospital mortality and that the duration of persistent lactic acidosis was more predictive of mortality than the initial lactate value [30]. Similarly, Friedman et al. showed in a 35patient study that survivors of severe sepsis admitted to the medical intensive care unit (MICU) or SICU had significantly lower lactate values at $24 \mathrm{~h}$ of resuscitation than nonsurvivors [31]. Finally, Manikis et al. followed lactate measurements every $8 \mathrm{~h}$ for $>72 \mathrm{~h}$ in 129 trauma patients and demonstrated serial lactate measurements and the duration of hyperlactemia to be reliable indicators of morbidity and mortality following trauma [32-34]. In a 94-patient SICU sepsis study, Marty et al. measured lactate at time $e_{0}(T), T_{6}, T_{12}$, and $T_{24}$ and showed that the best predictor of death was the $T_{24}$ clearance. These authors concluded that during the first $24 \mathrm{~h}$ in the ICU, hyperlactemia, even after the "golden hours," is associated with increased mortality, and lactate clearance-directed therapy should be considered for the first $24 \mathrm{~h}$ of treatment [36]. Similarly, in an 81-patient study, Herwanto et al. investigated the role of 6-, 12-, and 24-h lactate clearance in patients with sepsis and septic shock and found only the 24-h lactate clearance measurements to be associated with mortality [37].

\section{Rationale for further research}

Monitoring serial lactate measurements during early sepsis is a clinically useful tool as both a clinical end point to target and for prognostication; however, the utility of monitoring lactate beyond the initial $24 \mathrm{~h}$ of treatment is a poorly studied topic in the current literature.

Although late sepsis is loosely defined in prior studies, it is characterized by microcirculatory dysfunction leading to end organ failure and increased mortality $[11,12,15,38,39]$. Interestingly, failure to achieve lactate clearance beyond the golden hours despite microcirculatory restoration suggests that normalization of this microcirculatory dysfunction likely requires therapeutic interventions that differ from those used in early sepsis management [38, 40, 41]. Moreover, after the initial resuscitation has lead to restoration of microcirculatory parameters (i.e., cardiac output, $\mathrm{ScvO}_{2}$, mean arterial pressure), further attempts at normalizing lactate with traditional resuscitative techniques are likely detrimental $[11,12,15]$. For these reasons, further research evaluating the role of lactate kinetics during late sepsis management has the potential to guide physicians in their management of septic patients and improve clinical outcomes. Should an association between poor lactate kinetics and mortality exists during this later period, further studies could then investigate interventions targeting the microcirculatory impairment among septic patients with abnormal lactate kinetics after the first $24 \mathrm{~h}$ of resuscitation [38, 39].

Recently, the ProCESS, ARISE, and ProMISe trials have provided convincing evidence that EGDT is not superior to usual care in sepsis management [42-44]. With the foundational treatment paradigm for sepsis now being called into question, it is crucial to investigate the benefits of the individual components of sepsis management, both early and later in the course of care. Studies attempting to elucidate the potential value of one particular sepsis prognostic indicator, specifically lactate, later in the management of septic patients could prove beneficial [45].

\section{Conclusions}

Extensive investigations into the efficacy of lactate kinetics as a marker for response to resuscitative therapies in septic patients have demonstrated a clear association with clinical outcomes including mortality. However, the majority of these studies have focused on the utility during the early phase of sepsis management, with little attention regarding later time periods. Future studies focusing on lactate as a potential prognostic tool for late sepsis management and treatment duration have the potential to direct physicians in their care for septic patients during this understudied time period and improve patient outcomes.

\section{Competing interests}

The authors declare that they have no competing interests.

\section{Authors' contributions}

JC conceived the initial need for this review, conducted the initial literature review, and drafted the initial manuscript. MC helped draft the initial manuscript and conduct the initial literature review. BG served to provide major factual, organizational, and grammatical edits to the initial manuscript and helped devise and write subsequent drafts. JL participated in the design and coordination of the review and helped draft the initial manuscript and edit subsequent drafts. All authors read and approved the final manuscript.

\section{Acknowledgements}

There are no additional acknowledgements. None of the authors received any funding for this study.

\section{Author details}

${ }^{1}$ Department of Medicine, University of Florida College of Medicine, 1600 SW Archer Road, Gainesville, FL 32608, USA. Division of Pulmonary, Allergy, and Critical Care, University of Alabama-Birmingham, Birmingham, AL, USA.

${ }^{3}$ Division of Pulmonary Critical Care, University of Florida, Gainesville, FL, USA.

Received: 28 May 2015 Accepted: 25 September 2015

Published online: 06 October 2015

\section{References}

1. Mikkelsen ME, Miltiades AN, Gaieski DF, Goyal M, Fuchs BD, Shah CV, et al. Serum lactate is associated with mortality in severe sepsis independent of organ failure and shock. Crit Care Med. 2009;37:1670-7.

2. Nguyen HB, Rivers EP, Knoblich BP, Jacobsen G, Muzzin A, Ressler JA, et al. Early lactate clearance is associated with improved outcome in severe sepsis and septic shock. Crit Care Med. 2004;32:1637-42. 
3. Zimmerman JE, Kramer AA, Knaus WA. Changes in hospital mortality for United States intensive care unit admissions from 1988 to 2012. Crit Care. 2013;17:R81.

4. Stevenson EK, Rubenstein AR, Radin GT, Wiener RS, Walkey AJ. Two decades of mortality trends among patients with severe sepsis: a comparative meta-analysis*. Crit Care Med. 2014;42:625-31.

5. Bolton JD. Clinical use of lactate testing in shock states. Seminars in Anesthesia, Perioperative Medicine and pain. 2007:26:35-9.

6. D. DB. Lactic acidosis. Applied Physiology in Intensive Care Medicine 2012:111-114.

7. Gutierrez G, Wulf ME. Lactic acidosis in sepsis: a commentary. Intensive Care Med. 1996:22:6-16.

8. James JH, Luchette FA, McCarter FD, Fischer JE. Lactate is an unreliable indicator of tissue hypoxia in injury or sepsis. Lancet. 1999;354:505-8.

9. Levraut J, Ciebiera JP, Chave S, Rabary O, Jambou P, Carles M, et al. Mild hyperlactatemia in stable septic patients is due to impaired lactate clearance rather than overproduction. Am J Respir Crit Care Med. 1998;157:1021-6.

10. Gibot S. On the origins of lactate during sepsis. Crit Care. 2012;16:151.

11. van Genderen ME, Klijn E, Lima A, de Jonge J, Sleeswijk Visser S, Voorbeijtel J, et al. Microvascular perfusion as a target for fluid resuscitation in experimental circulatory shock. Crit Care Med. 2014;42:e96-e105.

12. Hernandez G, Luengo C, Bruhn A, Kattan E, Friedman G, Ospina-Tascon GA, et al. When to stop septic shock resuscitation: clues from a dynamic perfusion monitoring. Ann Intensive Care. 2014:4:30.

13. Marik P. Lactate clearance as a target of therapy in sepsis: a flawed paradigm. OA Crit Care. 2013;1:3.

14. Rivers EP, Elkin R, Cannon CM. Counterpoint: should lactate clearance be substituted for central venous oxygen saturation as goals of early severe sepsis and septic shock therapy? No. Chest. 2011;140:1408-13. discussion 1413-1409.

15. Bakker J. Lost in translation: on lactate, hypotension, sepsis-induced tissue hypoperfusion, quantitative resuscitation and surviving sepsis campaign bundles. Crit Care Med. 2015:43:705-6.

16. Jones AE. Lactate clearance for assessing response to resuscitation in severe sepsis. Acad Emerg Med. 2013;20:844-7.

17. Jones AE. Point: should lactate clearance be substituted for central venous oxygen saturation as goals of early severe sepsis and septic shock therapy? Yes. Chest. 2011;140:1406-8.

18. Bakker J, Nijsten MW, Jansen TC. Clinical use of lactate monitoring in critically ill patients. Ann Intens Care. 2013;3:12.

19. Dellinger RP, Carlet JM, Masur H, Gerlach H, Calandra T, Cohen J, et al. Surviving sepsis campaign guidelines for management of severe sepsis and septic shock. Intens Care Med. 2004;30:536-55.

20. Dellinger RP, Levy MM, Rhodes A, Annane D, Gerlach H, Opal SM, et al. Surviving sepsis campaign: international guidelines for management of severe sepsis and septic shock, 2012. Intens Care Med. 2013;39:165-228.

21. Trzeciak S, Dellinger RP, Chansky ME, Arnold RC, Schorr C, Milcarek B, et al. Serum lactate as a predictor of mortality in patients with infection. Intens Care Med. 2007;33:970-7

22. Nguyen HB, Loomba M, Yang JJ, Jacobsen G, Shah K, Otero RM, et al. Early lactate clearance is associated with biomarkers of inflammation, coagulation, apoptosis, organ dysfunction and mortality in severe sepsis and septic shock. J Inflamm. 2010;7:6.

23. Arnold RC, Shapiro NI, Jones AE, Schorr C, Pope J, Casner E, et al. Multicenter study of early lactate clearance as a determinant of survival in patients with presumed sepsis. Shock. 2009;32:35-9.

24. Jansen TC, van Bommel J, Schoonderbeek FJ, Sleeswijk Visser SJ, van der Klooster JM, Lima AP, et al. Early lactate-guided therapy in intensive care unit patients: a multicenter, open-label, randomized controlled trial. Am J Respir Crit Care Med. 2010;182:752-61

25. Jones AE, Shapiro NI, Trzeciak S, Arnold RC, Claremont HA, Kline JA. Lactate clearance vs central venous oxygen saturation as goals of early sepsis therapy: a randomized clinical trial. JAMA. 2010;303:739-46.

26. Otero RM, Nguyen HB, Huang DT, Gaieski DF, Goyal M, Gunnerson KJ, et al. Early goal-directed therapy in severe sepsis and septic shock revisited: concepts, controversies, and contemporary findings. Chest. 2006;130:1579-95.

27. Puskarich MA, Trzeciak S, Shapiro NI, Arnold RC, Heffner AC, Kline JA, et al. Prognostic value and agreement of achieving lactate clearance or central venous oxygen saturation goals during early sepsis resuscitation. Acad Emerg Med. 2012;19:252-8.
28. Puskarich MA, Trzeciak S, Shapiro NI, Albers AB, Heffner AC, Kline JA, et al. Whole blood lactate kinetics in patients undergoing quantitative resuscitation for severe sepsis and septic shock. Chest. 2013;143:1548-53.

29. Nguyen HB, Kuan WS, Batech M, Shrikhande P, Mahadevan M, Li CH, et al. Outcome effectiveness of the severe sepsis resuscitation bundle with addition of lactate clearance as a bundle item: a multi-national evaluation. Crit Care. 2011;15:R229.

30. Bakker J, Gris P, Coffernils M, Kahn RJ, Vincent JL. Serial blood lactate levels can predict the development of multiple organ failure following septic shock. Am J Surg. 1996;171:221-6.

31. Friedman G, Berlot G, Kahn RJ, Vincent JL. Combined measurements of blood lactate concentrations and gastric intramucosal ph in patients with severe sepsis. Crit Care Med. 1995:23:1184-93.

32. Manikis P, Jankowski S, Zhang H, Kahn RJ, Vincent JL. Correlation of serial blood lactate levels to organ failure and mortality after trauma. Am J Emerg Med. 1995;13:619-22

33. McNelis J, Marini CP, Jurkiewicz A, Szomstein S, Simms HH, Ritter G, et al. Prolonged lactate clearance is associated with increased mortality in the surgical intensive care unit. Am J Surg. 2001;182:481-5.

34. Krishna U, Joshi SP, Modh M. An evaluation of serial blood lactate measurement as an early predictor of shock and its outcome in patients of trauma or sepsis. Indian J Crit Care Med. 2009:13:66-73.

35. Husain FA, Martin MJ, Mullenix PS, Steele SR, Elliott DC. Serum lactate and base deficit as predictors of mortality and morbidity. Am J Surg. 2003;185:485-91.

36. Marty P, Roquilly A, Vallee F, Luzi A, Ferre F, Fourcade O, et al. Lactate clearance for death prediction in severe sepsis or septic shock patients during the first 24 hours in intensive care unit: an observational study. Ann Intensive Care. 2013;3:3.

37. Herwanto V. Role of 6-hour, 12-hour, and 24-hour lactate clearance in mortality of severe sepsis and septic shock patients. Crit Care. 2014;18.

38. Spronk PE, Zandstra DF, Ince C. Bench-to-bedside review: sepsis is a disease of the microcirculation. Crit care. 2004:8:462-8.

39. Saugel B, Trepte CJ, Heckel K, Wagner JY, Reuter DA. Hemodynamic management of septic shock: is it time for "individualized goal-directed hemodynamic therapy" and for specifically targeting the microcirculation? Shock. 2015;43:522-9.

40. Otto GP, Sossdorf M, Claus RA, Rodel J, Menge K, Reinhart K, et al. The late phase of sepsis is characterized by an increased microbiological burden and death rate. Crit Care. 2011;15:R183.

41. Rivers E, Nguyen B, Havstad S, Ressler J, Muzzin A, Knoblich B, et al. Early goal-directed therapy in the treatment of severe sepsis and septic shock. N Engl J Med. 2001;345:1368-77.

42. Lilly CM. The process trial-a new era of sepsis management. N Engl J Med. 2014;370:1750-1

43. Investigators A, Group ACT, Peake SL, Delaney A, Bailey M, Bellomo R, et al. Goal-directed resuscitation for patients with early septic shock. N Engl J Med. 2014:371:1496-506.

44. Pro Cl, Yealy DM, Kellum JA, Huang DT, Barnato AE, Weissfeld LA, et al. A randomized trial of protocol-based care for early septic shock. N Engl J Med. 2014;370:1683-93.

45. Zhang Z, Xu X. Lactate clearance is a useful biomarker for the prediction of all-cause mortality in critically ill patients: a systematic review and metaanalysis*. Crit Care Med. 2014;42:2118-25.

\section{Submit your next manuscript to BioMed Central and take full advantage of:}

- Convenient online submission

- Thorough peer review

- No space constraints or color figure charges

- Immediate publication on acceptance

- Inclusion in PubMed, CAS, Scopus and Google Scholar

- Research which is freely available for redistribution 